\title{
AORTOCORONARY CONDUITS MAY SHOW A DIFFERENT INFLAMMATORY RESPONSE - COMPARATIVE STUDY AT TRANSCRIPT LEVEL
}

Mariusz J. Nawrocki ${ }^{1}$, Sandra Kałużna², Karol Jopek ${ }^{2}$, Greg Hutchings ${ }^{1,3}$, Bartłomiej Perek ${ }^{4}$, Marek Jemielity ${ }^{4}$, Agnieszka Malińska, ${ }^{2,7}$ Bartosz Kempisty ${ }^{1,2,5,6}$, Paul Mozdziak ${ }^{8}$, Michał Nowicki ${ }^{2}$

\begin{abstract}
Coronary artery bypass grafting (CABG), together with percutaneous coronary intervention (PCI), are both still the most efficient procedures for myocardial revascularization to treat advanced coronary artery disease (CAD). Donor blood vessels used in CABG are usually the internal thoracic artery (ITA) and saphenous vein (SV). The importance of inflammation and inflammatory pathways in graft patency is well established. Nevertheless, not all molecular mechanisms underlying the inflammatory process appear to be clear. Employing the expressive microarray approach to analyze the transcriptome of both venous and arterial grafts, five GO BP terms has been selected: "cellular response to interferon-gamma", "inflammatory response", "interferon-gamma-mediated signaling pathway", "response to interferon-gamma" and "positive regulation of inflammatory response". This study aimed to evaluate potential molecular factors that could be characteristic markers for both SV and ITA conduits.
\end{abstract}

Running title: Aortocoronary conduits may show a different inflammatory response

Keywords: coronary artery bypass graftin, internal thoracic artery, saphenous vein, inflammation

\footnotetext{
${ }^{1}$ Department of Anatomy, Poznań University of Medical Sciences, Poznań, Poland

2Department of Histology and Embryology, Poznań University of Medical Sciences, Poznań, Poland

${ }^{3}$ The School of Medicine, Medical Sciences and Nutrition, University of Aberdeen, Aberdeen, UK

${ }^{4}$ Department of Cardiac Surgery and Transplantology, Poznań University of Medical Sciences, Poznań, Poland

${ }^{5}$ Department of Obstetrics and Gynecology, University Hospital and Masaryk University, Brno, Czech Republic

${ }^{6}$ Department of Veterinary Surgery, Institute of Veterinary Medicine, Nicolaus Copernicus University in Toruń, Toruń, Poland

${ }^{7}$ Division of Anatomy and Histology, University of Zielona Góra, Zielona Góra, Poland

${ }^{8}$ Physiology Graduate Program, North Carolina State University, Raleigh, North Carolina

* Correspondence: bkempisty@ump.edu.pl

Full list of author information is available at the end of article
} 


\section{Introduction}

Coronary artery bypass grafting (CABG) is still one of the most common surgical procedures aimed at improving blood circulation in atherosclerotic coronary arteries [1]. The predominant reason for early acute coronary syndromes is graft failure. Thus, better understanding of the activated molecular mechanisms occurring soon after surgery may further allow a reduction of the rate of these adverse cardiovascular events. There are a number of blood vessels which can be used as grafts in this revascularization procedure. Nevertheless, the internal thoracic artery (ITA) and the saphenous vein (SV) are the most commonly applied as aortocoronary conduits.

The early occlusion of ITA grafts is observed in approximately $5 \%$ of patients, whereas SV transplants occlude within a one year period after surgery in 10$15 \%$ of CABG patients [2]. Thus, venous conduits are commonly used in older patients. Despite advances in perioperative management and better understanding of the vessel wall histological architecture, current knowledge regarding molecular pathways' activation and their possible mechanisms in the perioperative period of CABG procedure is highly limited. There are several molecular pathways indicating increased inflammatory status, haemostasis activation, as well as increased oxidative stress, which seem to play a pivotal role in graft patency. Surgical stress accounts for more protracted and marked molecular pathway perturbations overall. Our previous molecular studies on ITA and SV grafts have shown that there are significant discrepancies between these vessels in the level of expression of genes involved in osteogenic changes in these vessels. According to our findings, we have suggested that venous grafts may be more exposed to atherosclerotic changes [3].

Bettering understanding of the activated molecular mechanisms occurring in vessels soon after surgery may allow a further reduction of the rate of adverse cardiovascular events. Therefore, our study aimed to investigate the transcriptomic profile of genes characterizing both ITA and SV. Employing the microarray approach, we analyzed differences at the molecular level between both blood vessels that often serve as aortocoronary grafts. We decided to additionally describe "cellular response to interferon-gamma", "inflammatory response", "interferon-gamma-mediated signaling pathway", "response to interferon-gamma" and "positive regulation of inflammatory response" gene ontology biological process (GO BP) terms, because inflammatory effects can affect the usefulness of all the blood vessels used as conduits in the CABG procedure.

\section{Materials and methods \\ Operation procedure and sample collection}

In most patients, the left ITA was used to bypass the left anterior descending coronary artery (LAD).
The other target coronary arteries were usually revascularized with SV grafts.

All surgeries were performed through median sternotomy. SV grafts were obtained through a full-length thigh incision over its course [4]. Pivotal points of the procedure included minimal manipulation of the graft ("no-touch" technique), avoiding extensive dilation of the conduits, using low-intensity electrocautery and the control of the branches with stainless-steel vascular clips. In all cases, the distal part of the obtained SV segment (at least $15-20 \mathrm{~mm}$ in length) was saved for further laboratory studies.

ITA conduits were harvested as pedicled, together with satellite veins and endothoracic fascia from the $2 \mathrm{nd}$ to 6 th intercostal space. The distal end of the ITA segment was divided at the level of its bifurcation. After heparinization, ITA conduits were clipped distally, injected with $10 \mathrm{~mL}$ of a papaverine solution $(1 \mathrm{mg} / \mathrm{mL})$, and allowed to pharmacologically dilate. Immediately before anastomosis of the distal end of ITA to the recipient coronary artery, a $10-\mathrm{mm}$ segment of the conduit was harvested for further molecular and histological tests.

The sets of the vessel samples, both SV and ITA, were immediately snap-frozen in liquid nitrogen and stored at $-80{ }^{\circ} \mathrm{C}$ until RNA isolation. Another set of samples was directed for histochemical examination. Transcriptome screening analysis was performed on $18 \mathrm{SV}$ and 20 ITA samples.

\section{Microarray expression study and data analysis}

Our experiment employed 38 GeneChip ${ }^{\circledR}$ HGU219 (Affymetrix, Santa Clara, CA, USA) microarrays to simultaneously examine thousands of transcripts for each of the analyzed samples. In the first step, the total RNA (500 ng) from each pooled sample was subjected to two rounds of sense cDNA amplification (Ambion ${ }^{\circledR}$ WT Expression Kit, provided by Ambion, Austin, TX, USA). The synthesis of cRNA was performed by in vitro transcription $(16 \mathrm{~h}, 40$ $\left.{ }^{\circ} \mathrm{C}\right)$. Then, cRNA was purified and re-transcribed into cDNA. Subsequently, cDNA samples were used for biotin labeling and fragmentation using an Affymetrix GeneChip ${ }^{\circledR}$ WT Terminal Labeling and Hybridization kit (Affymetrix). Next, the biotin-labeled samples were loaded onto and hybridized to the Affymetrix ${ }^{\circledR}$ Human Genome U219 Array Strip. Hybridization was conducted at $48^{\circ} \mathrm{C}$ for $20 \mathrm{~h}$, employing an AccuBlock ${ }^{\mathrm{TM}}$ Digital Dry Bath (Labnet International, Inc., Edison, NJ, USA) hybridization oven. Then, microarrays were washed and stained, according to technical protocol, using an Affymetrix GeneAtlas ${ }^{T M}$ Fluidics Station (Affymetrix, Santa Clara, CA, USA). The strips were scanned using an Affymetrix GeneAtlas ${ }^{\mathrm{TM}}$ Imaging Station (Affymetrix, Santa Clara, CA, USA). The scans of the microarrays were saved on hard drives as *.CEL files for downstream data analysis. 
Quality control (QC) studies were performed using the Affymetrix GeneAtlas ${ }^{\mathrm{TM}}$ Instrument Control Software 2.0.0.460 (Affymetrix, Santa Clara, CA, USA), according to the manufacturer's standards. The generated *.CEL files were subjected to further analysis performed using the R statistical language and Bioconductor package with the relevant Bioconductor libraries. To correct the background, normalize, and summarize the results, we used the robust multiarray averaging (RMA) algorithm. Assigned biological annotations were obtained from the "pd.ragene.2.1.st" library and employed for the mapping of normalized gene expression values with
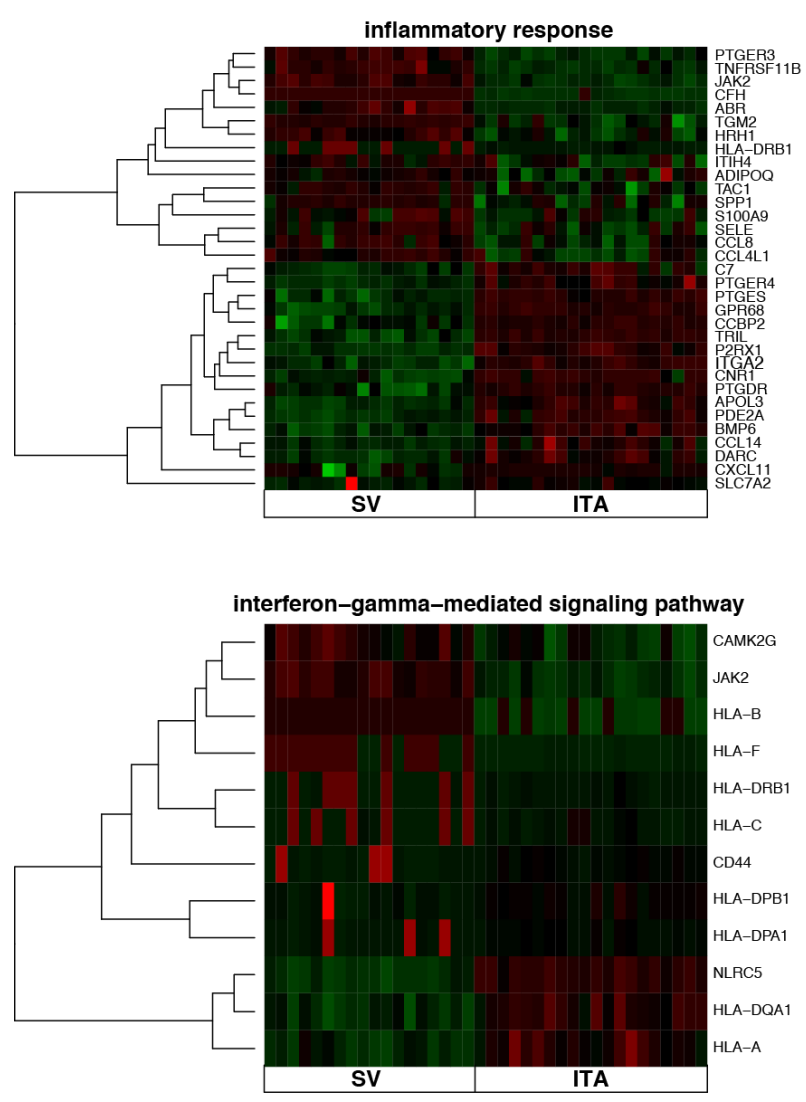

their symbols, gene names, and Entrez IDs, allowing generation of a complex gene data table. To determine the statistical significance of the analyzed genes, moderated t-statistics from the empirical Bayes method were performed. The obtained p-values were corrected for multiple comparisons using Benjamini and Hochberg's false discovery rate and described as adjusted p-values. The selection of significantly altered genes was based on a p-value beneath 0.05 and an expression higher than two-fold. The differentially expressed gene list (separated for upregulated and downregulated genes) was uploaded to the DAVID Bioinformatics Resources 6.8
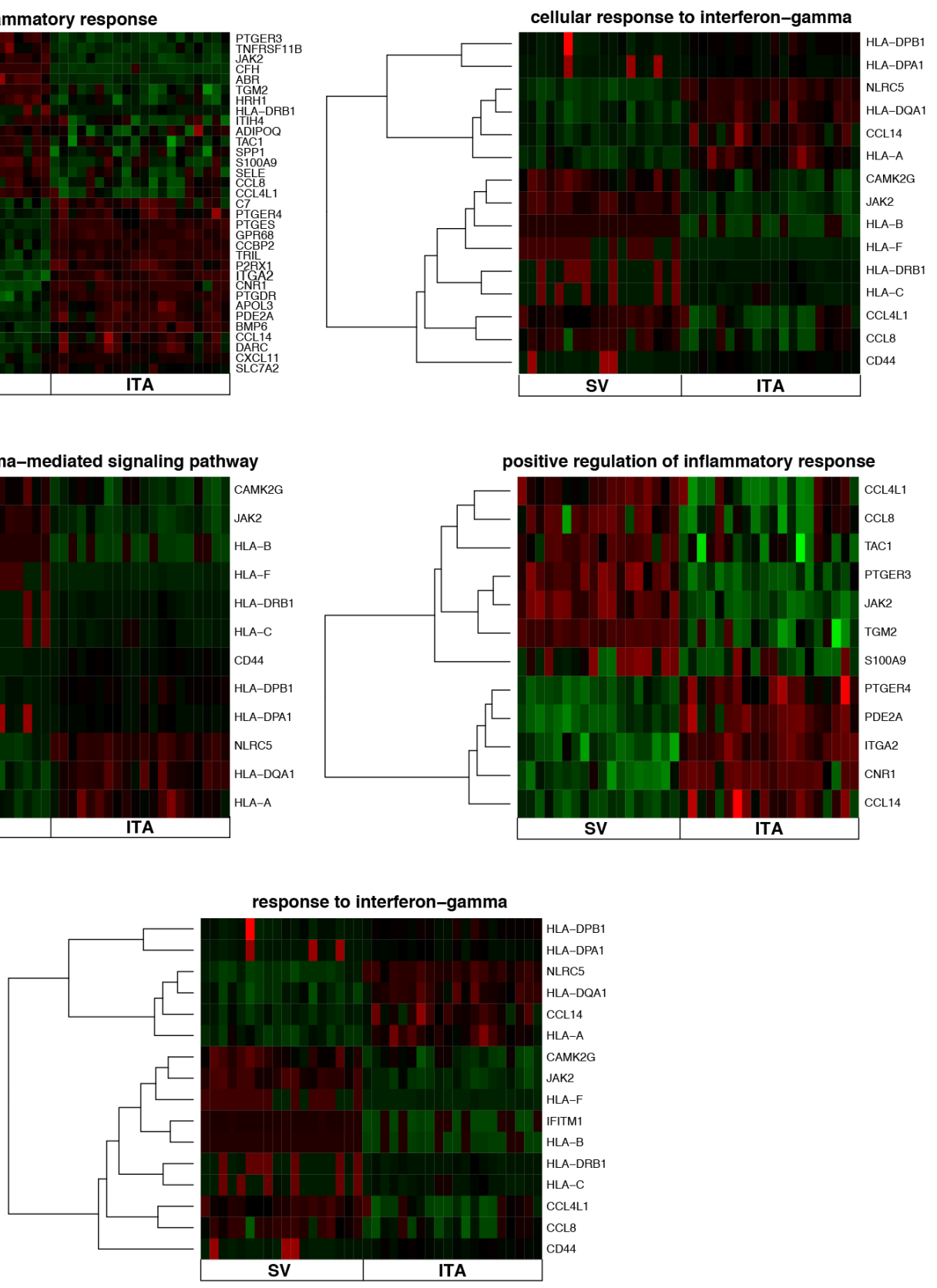

FIGURE 1 Heatmaps presenting differentially expressed genes involved in "cellular response to interferon-gamma", "inflammatory response", "interferon-gamma-mediated signaling pathway", "response to interferon-gamma" and "positive regulation of inflammatory response" based on GO BP terms. Each row on the Y axis represents a single transcript. The red color indicates downregulated genes while the green are upregulated 


\begin{tabular}{|c|c|c|c|}
\hline Gene symbol & Gene name & Fold change & Adj. p.val \\
\hline TNFRSF11B & tumor necrosis factor receptor superfamily, member $11 \mathrm{~b}$ & 4.74 & $<0.01$ \\
\hline CCL4L1 & chemokine ( $\mathrm{C}-\mathrm{C}$ motif) ligand 4 -like 1 & 4.53 & $<0.01$ \\
\hline SELE & selectin $\mathrm{E}$ & 3.76 & 0.01 \\
\hline PTGER3 & prostaglandin E receptor 3 (subtype EP3) & 3.53 & $<0.01$ \\
\hline CCL8 & chemokine ( $\mathrm{C}-\mathrm{C}$ motif) ligand 8 & 3.23 & $<0.01$ \\
\hline JAK2 & Janus kinase 2 & 3.18 & $<0.01$ \\
\hline TAC1 & tachykinin, precursor 1 & 3.17 & $<0.01$ \\
\hline S100A9 & S100 calcium binding protein $\mathrm{A} 9$ & 3.15 & 0.02 \\
\hline SPP1 & secreted phosphoprotein 1 & 2.97 & $<0.01$ \\
\hline TGM2 & transglutaminase 2 (C polypeptide, protein-glutamine-gamma-glutamyltransferase) & 2.58 & $<0.01$ \\
\hline CCL14 & chemokine ( $\mathrm{C}-\mathrm{C}$ motif) ligand 14 & -2.43 & $<0.01$ \\
\hline BMP6 & bone morphogenetic protein 6 & -2.45 & $<0.01$ \\
\hline $\mathrm{CFH}$ & complement factor $\mathrm{H}$ & -2.63 & $<0.01$ \\
\hline CD44 & CD44 molecule (Indian blood group) & -2.73 & $<0.01$ \\
\hline $\mathrm{C} 7$ & complement component 7 & -2.78 & $<0.01$ \\
\hline TRIL & TLR4 interactor with leucine-rich repeats & -2.95 & $<0.01$ \\
\hline PDE2A & phosphodiesterase 2A, cGMP-stimulated & -2.98 & $<0.01$ \\
\hline NLRC5 & NLR family, CARD domain containing 5 & -3.16 & $<0.01$ \\
\hline CNR1 & cannabinoid receptor 1 (brain) & -3.27 & $<0.01$ \\
\hline $\mathrm{P} 2 \mathrm{RX} 1$ & purinergic receptor $\mathrm{P} 2 \mathrm{X}$, ligand-gated ion channel, 1 & -6.48 & $<0.01$ \\
\hline
\end{tabular}

TABLE 1 The 10 most significantly upregulated and 10 most significantly downregulated genes involved in inflammatory response

software (Database for Annotation, Visualization and Integrated Discovery) [5], where the significantly upregulated Gene Ontology (GO) terms were extracted. The selection of significantly altered GO terms was based on a p-value (Benajamini) $<0.05$ and the volume of at least five genes.

To further investigate the chosen gene sets, we investigated their mutual relations with the GOplot package [6]. Subsequently, sets of differentially expressed genes from selected GO BP terms were applied to the STRING10 software (Search Tool for the Retrieval of Interacting Genes/Proteins) for interactions prediction. STRING is a huge database containing information on protein/gene interactions, including experimental data, computational prediction methods, and public text collections.

\section{Ethical approval}

The research related to human use has been complied with all the relevant national regulations, institutional policies and in accordance the tenets of the Helsinki Declaration, and has been approved by the authors' institutional review board or equivalent committee. Bioethical Committee approval no. $1201 / 08$, approved on $18 / 12 / 2008$.

\section{Results}

We used Human Genome U219 Array Strip for the microarray gene expression analysis of internal thoracic artery (ITA) and the saphenous vein (SV). This method allowed us to study the gene expres- sion of 49,308 transcripts. We selected genes with more than 2- fold changes and corrected p-values less than 0.05 for downstream analysis. A total of 1170 differentially expressed genes (DEGs) were identified according to the above criteria. We started the microarray gene expression analysis with subjecting the list of DEGs to DAVID software, which showed that the genes can be assigned to many gene ontology groups (GO BP terms). This paper focused on the genes involved in inflammatory response. The DAVID software indicated the following GO BP terms, which cover the above processes: "cellular response to interferon-gamma", "inflammatory response", "interferon-gamma-mediated signaling pathway", "response to interferon-gamma" and "positive regulation of inflammatory response". The 44 genes involved in those processes were clustered using hierarchical clustering and presented as heatmaps (Fig. 1).

It is worth mentioning that 29 genes were downregulated while 15 genes were upregulated. The 10 most significantly upregulated and downregulated genes, their symbols, fold changes and corrected pvalues are shown in table 1.

In the next part of analysis, we focused on the z-scores, which tell us whether the biological process is more likely to be decreased (negative value) or increased (positive value). The z-scores were presented as bar plot (Fig. 2) and as segments of inner circles in the figure 3 . As can be seen from the figures, only "positive regulation of inflamma- 


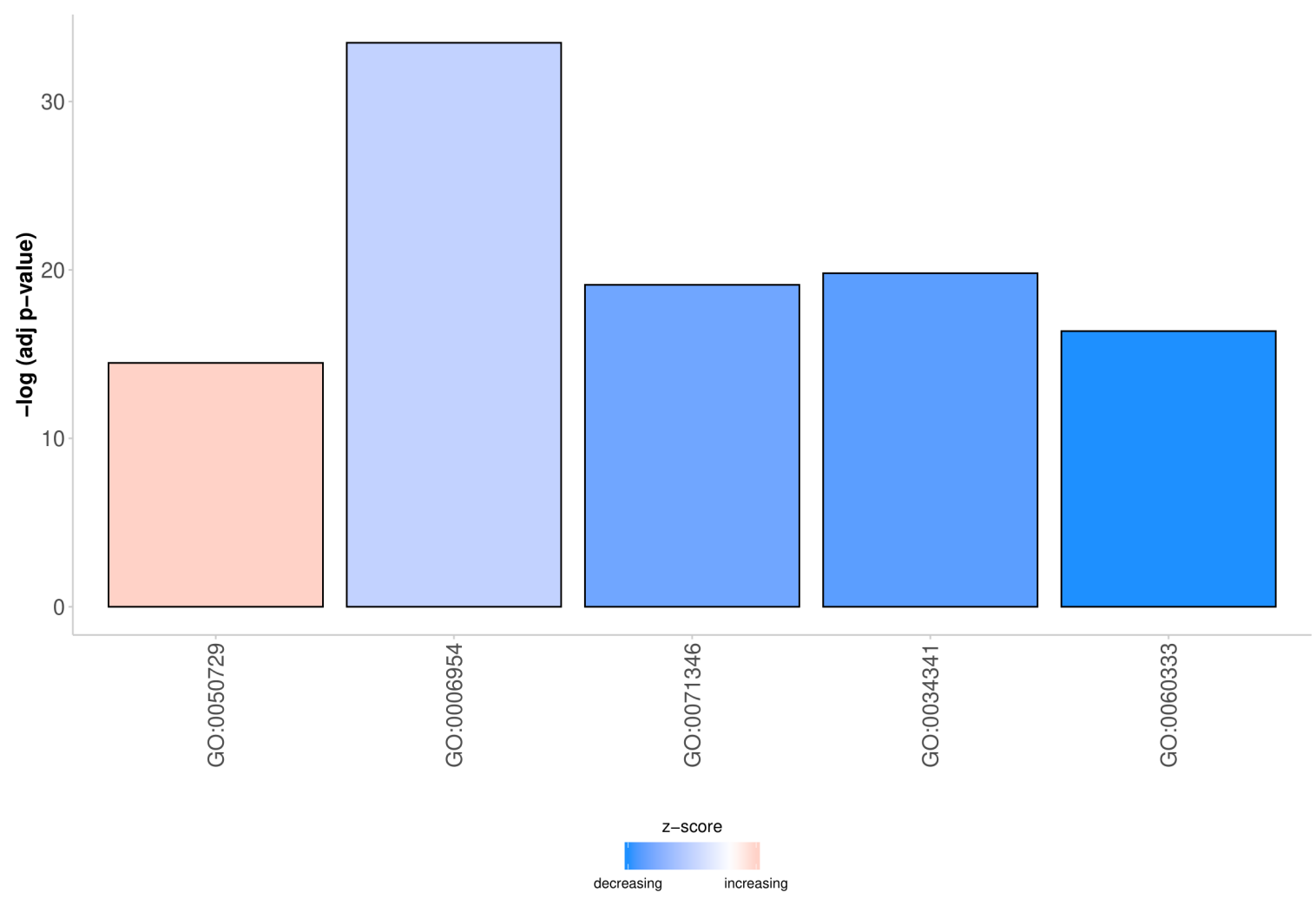

FIGURE 2 The bar plot presenting z-scores of processes involved in inflammatory response

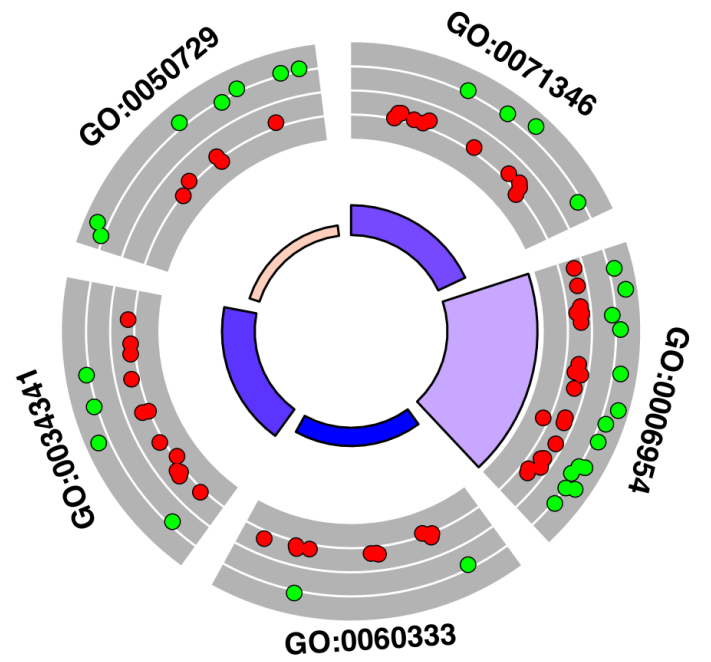

\begin{tabular}{|c|r|}
\hline ID & \multicolumn{1}{|c|}{ Description } \\
\hline GO:0071346 & cellular response to interferon-gamma \\
\hline GO:0006954 & inflammatory response \\
\hline GO:0060333 & interferon-gamma-mediated signaling pathway \\
\hline GO:0034341 & response to interferon-gamma \\
\hline GO:0050729 & positive regulation of inflammatory response \\
\hline
\end{tabular}

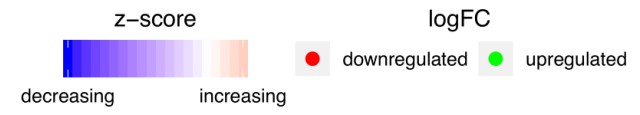

FIGURE 3 The circular scatter plots of differentially expressed genes involved in "cellular response to interferon-gamma", "inflammatory response", "interferon-gamma-mediated signaling pathway", "response to interferon-gamma" and "positive regulation of inflammatory response" GO BP terms. Each dot represents a single gene. The z-scores were presented as segments of inner circles

tory response" GO BP term is upregulated while the other biological processes are downregulated.

In the next section, we checked the interaction between selected ontological groups. One of the most visually appealing way of presenting such interaction is dendrogram (Fig. 4). Clusters contain functionally related genes based on their expression pattern. The middle circle represents a logarithm of 


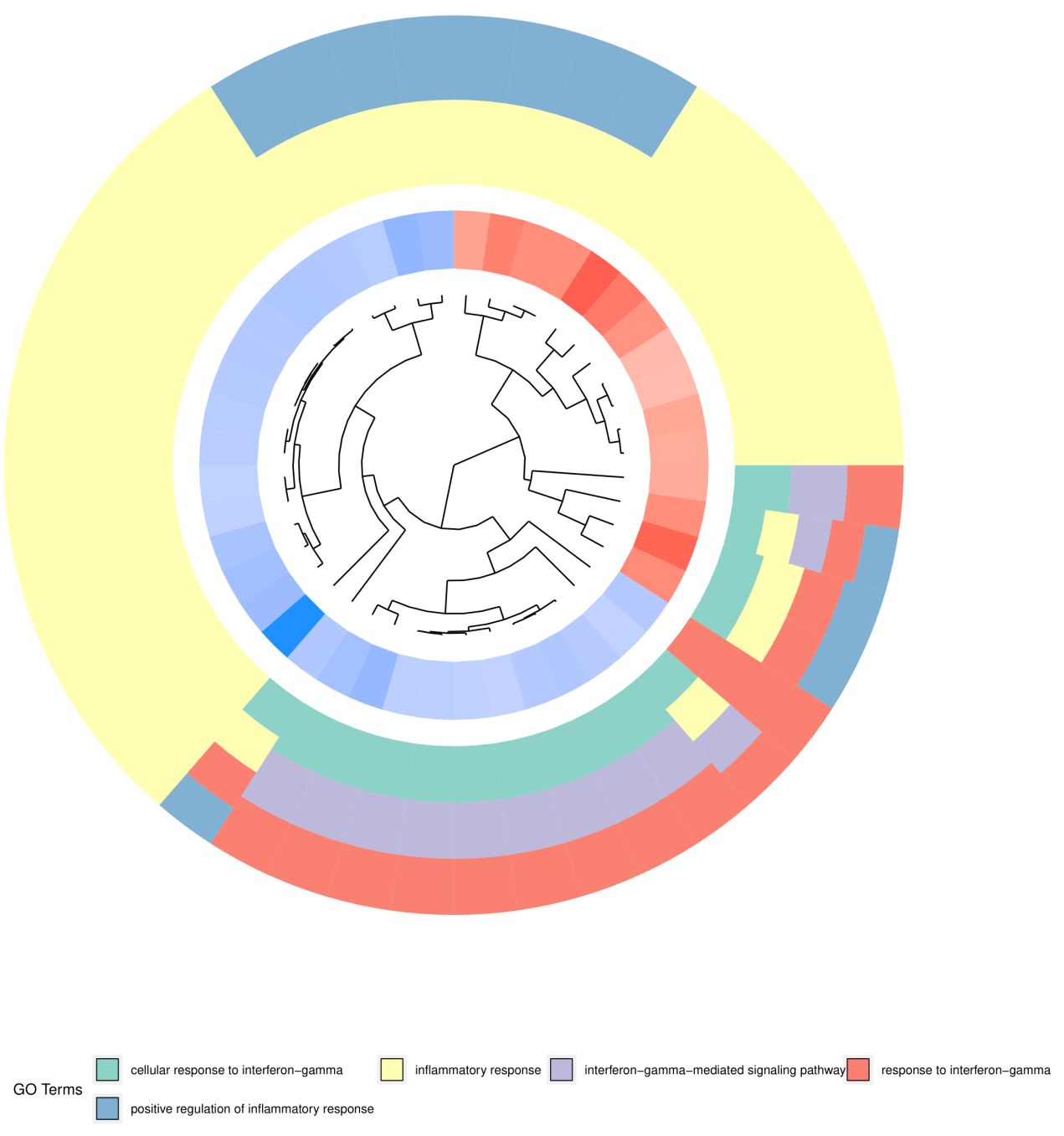

FIGURE 4 The dendrogram of differentially expressed genes involved in "cellular response to interferon-gamma", "inflammatory response", "interferon-gamma-mediated signaling pathway", "response to interferon-gamma" and "positive regulation of inflammatory response" GO BP terms. The DEGs were clustered based on their logFC values

fold change (logFC) of differentially expressed genes assigned to the studied GO terms. The GO terms are shown as the outer ring. The genes whose expression is downregulated form clusters marked by blue part of the middle circle and analogously, red indicates upregulated genes. Clusters of the same color over the entire width of the outer circle represent genes that are unique for a specific GO term. Clusters of different colors on the cross section of outer circle show sets of genes which are likely to be functionally related. The dendrogram showed that many genes belong simultaneously to "cellular response to interferon-gamma", "interferon-gamma-mediated signaling pathway" and "response to interferon-gamma". The genes that are unique for a specific GO term belong mainly to "inflammatory response".

In the gene ontology database, single genes may belong to many ontological terms. For this reason, we used plots with visualization of $\log F C$ values and relationship between genes and selected GO BP terms (Fig. 5). The relationship was also presented as a heatmap (Fig. 6). The strongest upregulated genes from examined GO BP terms included, among others: CCL4L1- C-C Motif Chemokine Ligand 4 Like 1, CCL8C-C Motif Chemokine Ligand 8 and JAK2- Janus kinase 2 . The most downregulated gene is P2RX1-purinergic receptor $\mathrm{P} 2 \mathrm{X}$, ligand-gated ion channel, 1.

In the next part of analysis, we focused on the interaction between proteins encoded by DEGs belonging to studied GO BP terms. Firstly, we used STRING software for the interaction prediction (Fig. 7).

Finally, we used ReactomeFIViz app for investigation of functional interactions between proteins encoded by DEGs belonging to selected GO BP terms. Among the most significantly enriched functional interaction networks were FI networks for "Immune 


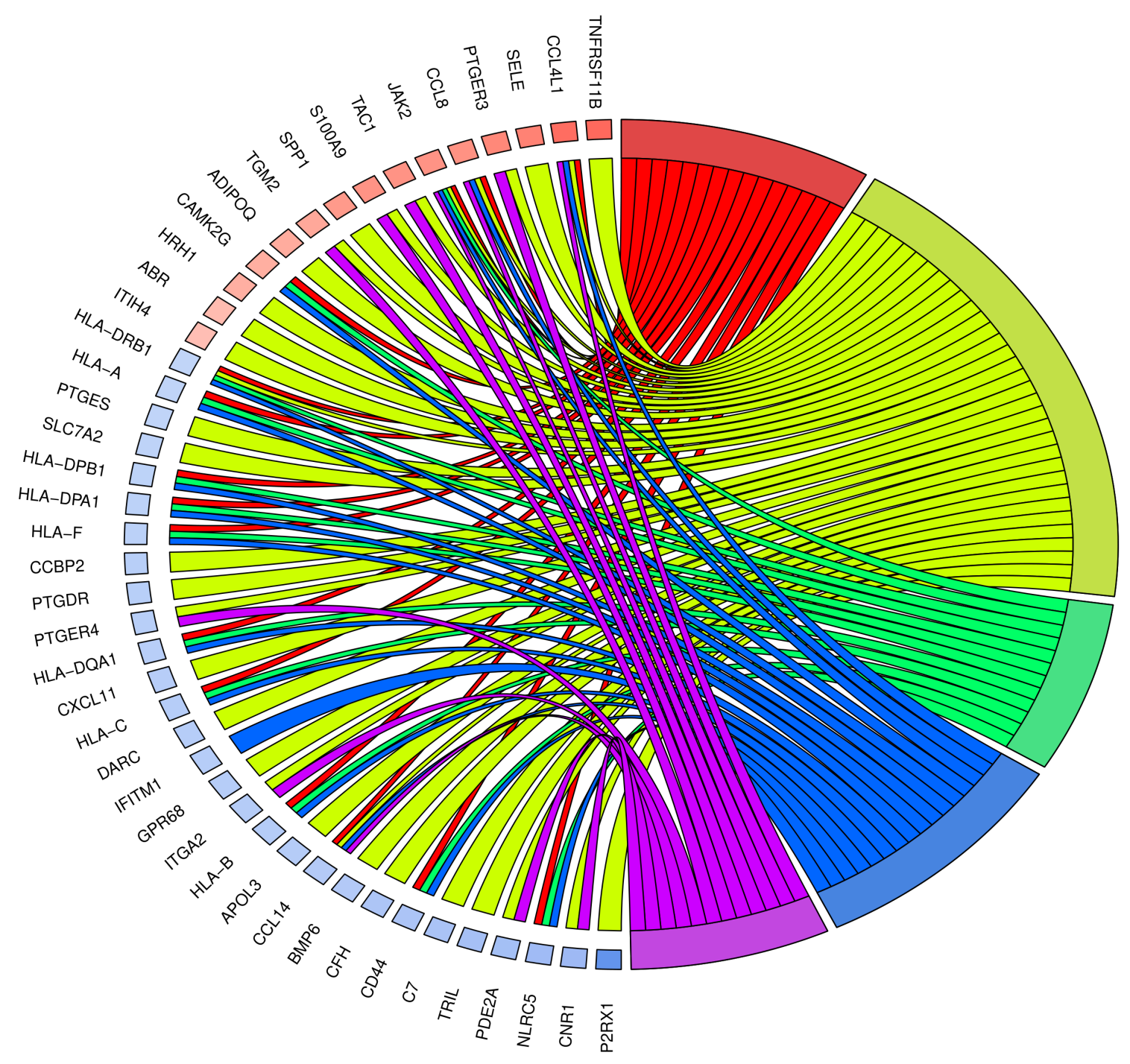

FIGURE 5 Analysis of enriched gene ontological groups involved in inflammatory response. The network plot presenting the linkages of genes and GO BP terms

response to interferon-gammapositive regulation of inflammatory responseinterferon-gamma-mediated signaling pathway cellular response to interferon-gamma-

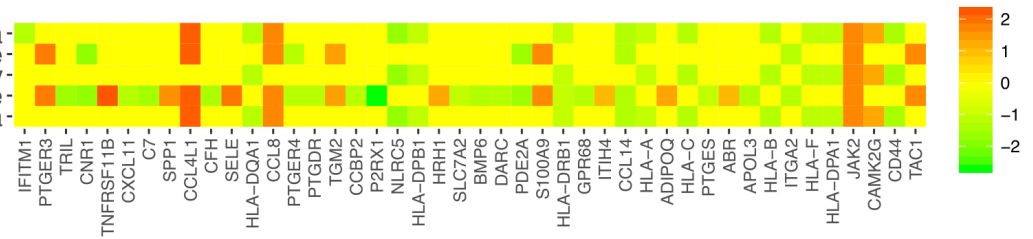

FIGURE 6 Heatmap presenting the relationship between genes and selected GO BP terms. The yellow color of tiles indicates the absence of $\operatorname{logFC}$ values

response" and "Interferon-gamma-mediated signaling pathway" (Fig. 8 and 9).

\section{Discussion}

While CABG has been performed for decades, questions remain regarding the detection and characterization of potential markers that will be valu- able to evaluate the conduits patency used in this procedure. The predominant cause of early acute coronary syndromes (ACS) is graft failure. Thus, better understanding of the activated molecular mechanisms occurring soon after surgery may allow a further reduction of the rate of these adverse cardiovascular events. The importance of inflamma- 


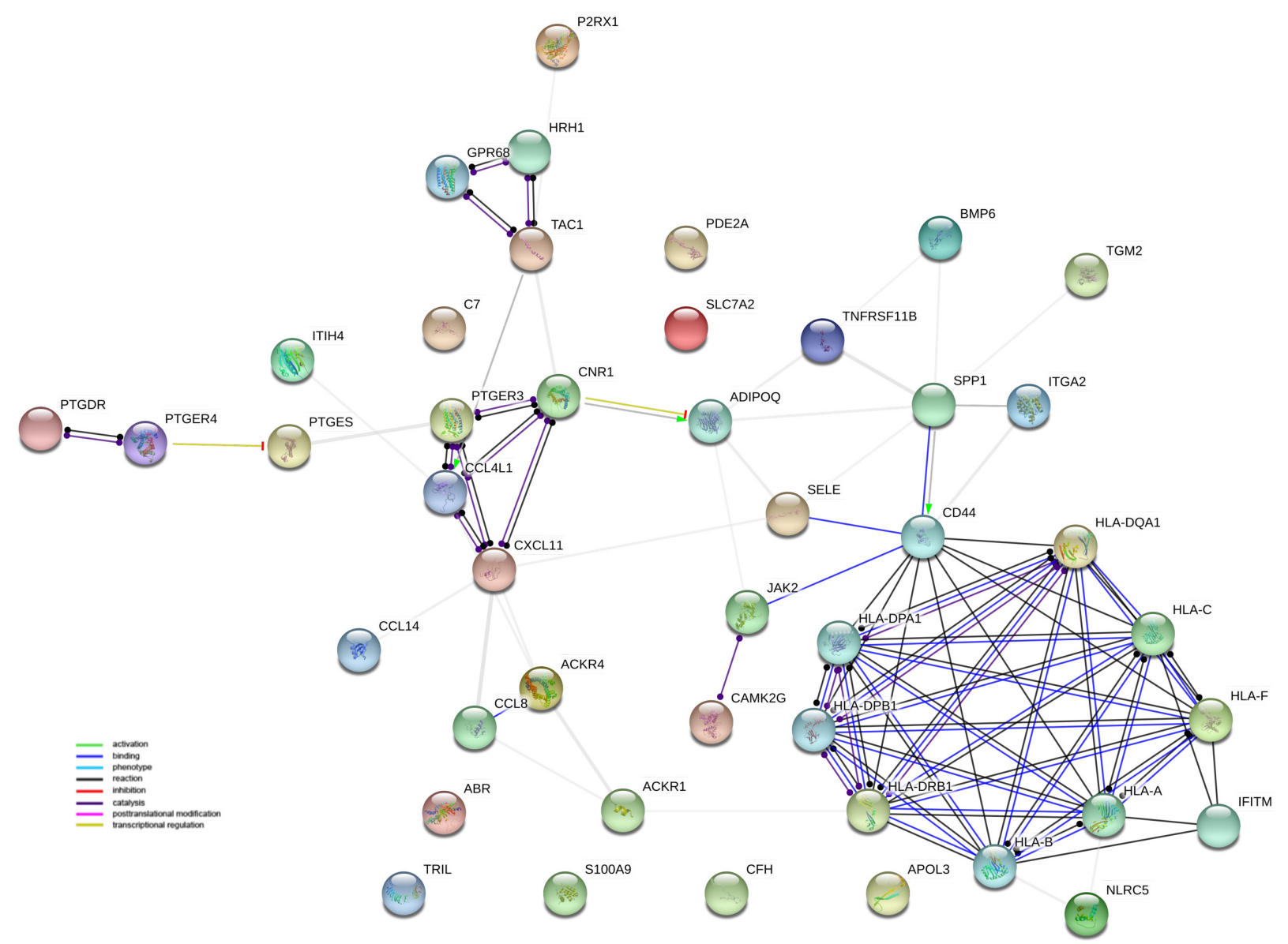

FIGURE 7 Interaction network of proteins encoded by DEGs belonging to "cellular response to interferon-gamma", "inflammatory response", "interferon-gamma-mediated signaling pathway", "response to interferon-gamma" and "positive regulation of inflammatory response" GO BP terms. The network was generated by STRING software. Network nodes represent proteins. Empty nodes indicate proteins of unknown 3D structure

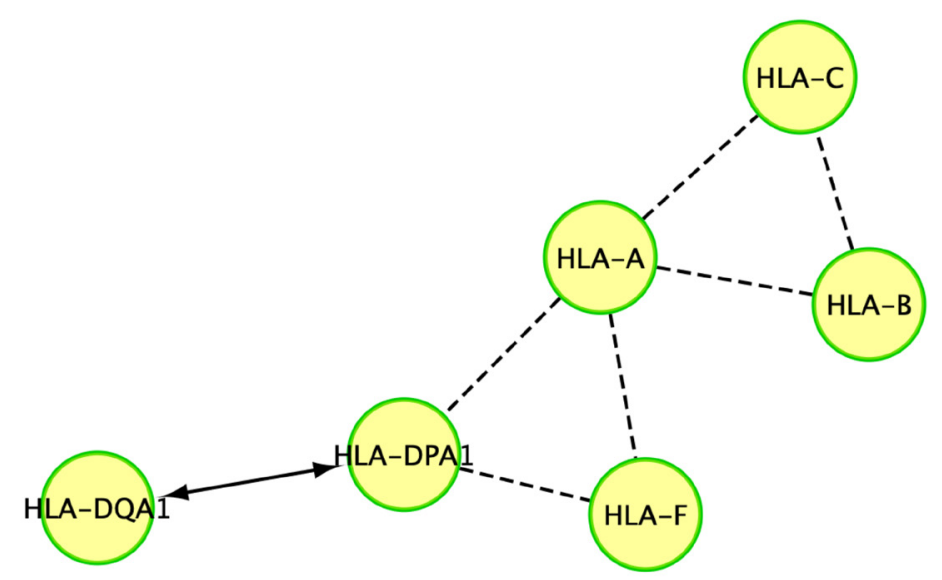

CXCL11

FIGURE 8 Reactome FI network for "Immune response". “--->” indicates activating/catalyzing, "-“ FIs extracted from complexes or inputs and "---" predicted FIs 


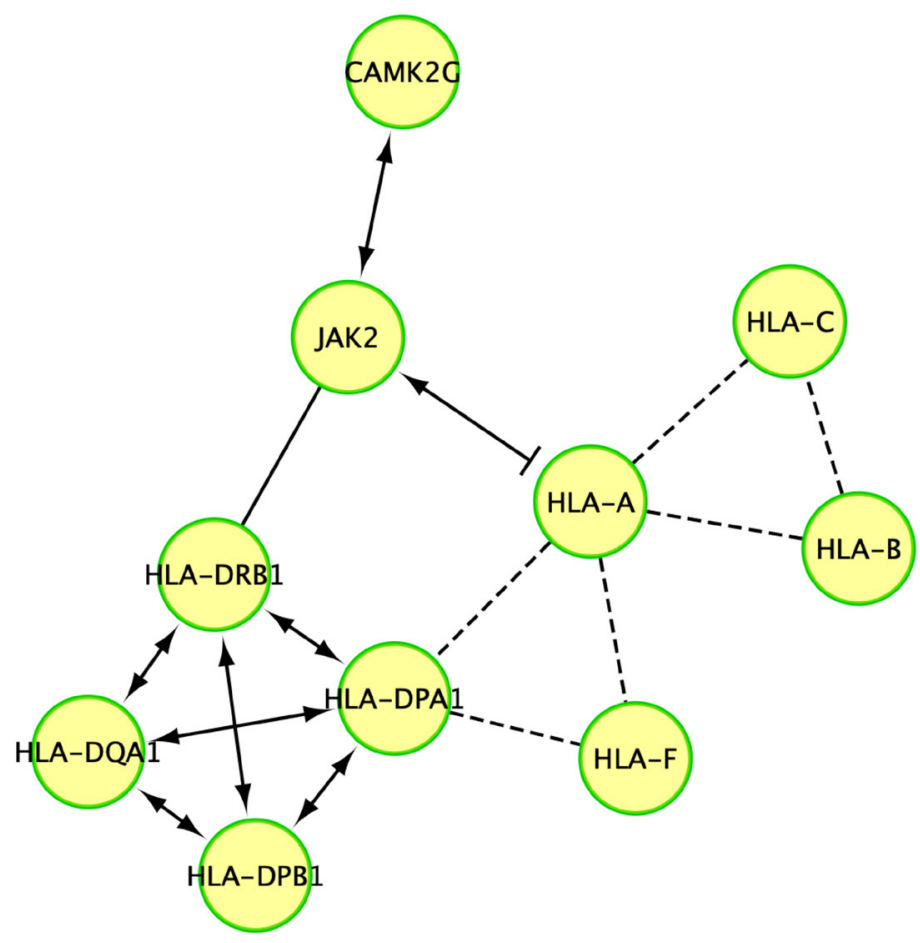

FIGURE 9 Reactome FI network for "Interferon-gamma-mediated signaling pathway". “--->” indicates activating/catalyzing, "-|" for inhibition, "-“ FIs extracted from complexes or inputs and "---" predicted FIs

tion and inflammatory pathways in atherosclerotic disease and ACS is well established. Nevertheless, not all molecular mechanisms underlying the inflammatory process appear to be clear. Thus, the present study aimed to compare the level of expression of genes involved in inflammatory processes in both ITA and SV conduits and describe potential molecular factors for the evaluation of ITA and SV segment quality.

Employing the expressive microarray approach to analyze the transcriptome of both venous and arterial grafts, five GO BP terms have been selected: "cellular response to interferon-gamma", "inflammatory response", "interferon-gamma-mediated signaling pathway", "response to interferon-gamma" and "positive regulation of inflammatory response". Among all selected ontological groups genes involved in the formation and maintenance of inflammatory processes can be distinguished. Overall, the genes presented on heatmaps showed differential expression patterns in both analyzed vessels.

Our results indicate the highest fold change of TNFRSF11B transcript levels from all differentially expressed genes analyzed in this study. Our data indicates higher TNFRSF11B transcript expression levels in ITA. Tumor necrosis factor receptor superfamily member $11 \mathrm{~b}$ (TNFRSF11B, other name: osteoprotegerin (OPG)) is a glycoprotein that acts as a cytokine of the tumor necrosis factor (TNF) [7]. In mice model without expression of OPG authors have shown increased calcification of the aortic media, particularly when given a high dose of phosphate or vitamin D3. These arteries are also the sites of endogenous OPG expression in normal arteries, raising the possibility of a protective role of OPG. This protective role of OPG is particularly important for preventing vascular calcification occurring secondary to administration of warfarin and vitamin D3 [8]. A potential protective effect of OPG against dangerous rupture in advanced abdominal aortic calcification development has been described [9]. Interestingly, in human studies, a significant association between levels of circulating OPG and vascular calcification has been shown. The presence of abdominal aortic calcification, a known risk factor in the development of abdominal aortic aneurysms, was weakly associated with the progression of abdominal aortic aneurysms [10]. Other studies suggested that a pathological increase of serum TNFRSF11B levels might play an important role in promoting leukocyte/endothelial cell adhesion and endothelial cells dysfunction [11]. In the latest scientific reports authors demonstrated that plasma TNFRSF11B levels were significantly associated with the presence of obstructive sleep apnea (OSA) and its severity. Plasma TNFRSF11B levels provided higher discriminatory accuracy levels for patients with OSA. TNFRSF11B could be a biomarker with a positive diagnostic value for premature vascular endothelial dysfunction in patients with OSA [12].

Transcript levels of $\mathrm{C}$-C chemokine ligand 4-like 1 (CCL4L1) also showed one of the highest fold change value among the genes analyzes, involved in the inflammatory process. Similar to TNFRSF11B 
transcripts, we observed higher expression levels of CCL4L1 mRNA in arterial conduits. This gene is one of several cytokine genes (together with CCL3-CCL3L1 and CCL4) that are clustered on the q-arm of chromosome 17 [13]. During emergency repair of ruptured abdominal aortic aneurysms, researchers collected aortic wall samples and investigated gene expression using microarrays [14]. The obtained results pointed to overexpression of a set of genes, including CCL4L1. High expression of CCL4L1 in end-stage of AAA disease may identify CCL $4 L 1$ as candidate gene related to processes involved in fibrosis. Studies analyzed 127 kidney biopsies obtained from HLA-sensitized (HS), non-HS patients and control individuals, and have shown CCL4L1 expression activated by antibody-dependent cellular cytotoxicity (ADCC) [15].

It is also worth noting that our results indicate a very significant participation of the human leukocyte antigen (HLA) gene complex encoding the major histocompatibility complex (MHC) proteins. We found 4 of the HLA Class I members (HLA-A, HLA-B, $H L A-C$ and $H L A-F$ ) and 4 belonging to HLA class II transcripts (HLA-DRB1, HLA-DPB1, HLA-DPA1 and $H L A-D Q A 1)$. These, highly polymorphic, cell-surface proteins are responsible for the regulation of the immune system in humans. Class I molecules play a central role in the immune system by presenting peptides derived from the endoplasmic reticulum lumen, while class II molecules are recognized by receptors on CD4-positive helper $\mathrm{T}$ cells when attacking and eliminating foreign substances [16]. Studies examining the pathogenesis of abdominal aortic aneurysm (AAA) have shown that both HLA-A and $H L A-B$ may be important genetic risk factors for the development of this abdominal aorta pathology [17]. Other studies indicated that bacterial lipopolysaccharide (LPS) enhanced expression of $H L A-A, H L A-B$ and HLA-C in cultured human endothelial cells, whereas it inhibits $H L A-D R$ molecule expression [18]. The new approach to avoid the risk of severe adverse reactions of small diameter vascular grafts may employ HLA-matched vascular grafts. Mallis et al. proposed a solution of the limited availability of the vessels by the use of HLAmatched vascular grafts utilizing the decellularized human umbilical arteries [19]. Our results did not show one expression pattern for HLA gene complex. Most members of the HLA family showed higher transcript levels in arterial conduits, while HLA-A and HLA-DQA1 were upregulated in SV samples.

The most significantly downregulated genes was purinergic receptor $\mathrm{P} 2 \mathrm{X} 1$ (P2RX1). The protein encoded by this gene belongs to the P2X family of G-protein-coupled receptors [20]. Next Generation Sequencing studies analyzing epifascial lymphatic collectors of the thigh, which were isolated for autologous transplantations, found therapeutic targets, suitable for acceleration of lymphatic contractility. Among the most promising results, the authors mention $P 2 R X 1$ as potential target for diagnosis and therapy [21]. Hennigs et al. [22] showed strong pulmonary endothelial staining of P2XR1, which can contribute to an unfavorable pulmonary disease phenotype found in pulmonary arterial hypertension. We found higher transcript levels of this gene in saphenous vein.

\section{Conclusions}

Our transcriptomic analysis of two conduits most commonly applied in coronary artery bypass grafting procedure, the internal thoracic artery (ITA) and the saphenous vein (SV), showed potential molecular markers of formation and maintenance of inflammatory changes. We hope that an analysis of the expression of genes involved in inflammatory response may help to identify patients at high risk of grafts occlusion.

\section{Acknowledgements}

This publication is part of the project: (1) "WroVasc-Integrated Cardiovascular Centre", co-financed by the European Regional Development Fund, within Innovative Economy Operational Program, 2007-2013 realized in Regional Specialized Hospital, Research and Development Center in Wroclaw.

\section{Corresponding author}

Corresponding author: Bartosz Kempisty PhD, Department of Histology and Embryology, Department of Anatomy, Poznań University of Medical Sciences, 6 Święcickiego St., 60-781 Poznań, Poland Tel./Fax: +48 618546418 / +48 61 8546440, e-mail: bkempisty@ ump.edu.pl.

\section{Conflict of interest statement}

The authors declare they have no conflict of interest.

\section{References}

1. Kappetein AP, van Mieghem NM, Head SJ. Revascularization Options. He art Fail Clin. 2016;12:135-9; DOI:10.1016/j.hfc.2015.08.011.

2. Davierwala PM, Mohr FW. Bilateral internal mammary artery grafting: Rationale and evidence. Int J Surg. 2015;16:133-9; DOI:10.1016/J. IJSU.2015.01.012

3. Nawrocki MJ, Perek B, Sujka-Kordowska P, Konwerska A, Kałużna S, Zawierucha P, Bruska M, Zabel M, Jemielity M, Nowicki M, Kempisty B, Malińska A. Differences in expression of genes involved in bone development and morphogenesis in the walls of internal thoracic artery and saphenous vein conduits may provide markers useful for evaluation graft patency. Int J Mol Sci. 2019;20; DOI:10.3390/ijms20194890.

4. Nasso G, Anselmi A, Modugno P, Alessandrini F. Minimally invasive saphenous vein harvesting guided by preoperative echotomography: results of a prospective randomized study. Interact Cardiovasc Thorac Surg. 2005;4:464-8; DOI:10.1510/icvts.2005.107854.

5. Huang DW, Sherman BT, Tan Q, Kir J, Liu D, Bryant D, Guo Y, Stephens R, Baseler MW, Lane HC, Lempicki RA. DAVID Bioinformatics Resources: expanded annotation database and novel algorithms to better extract biology from large gene lists. Nucleic Acids Res. 2007;35:W169-75; DOI:10.1093/nar/gkm415.

6. Walter W, Sánchez-Cabo F, Ricote M. GOplot: an R package for visually combining expression data with functional analysis: Fig. 1. Bioinformatics. 2015;31:2912-4; DOI:10.1093/bioinformatics/btv300.

7. Venuraju SM, Yerramasu A, Corder R, Lahiri A. Osteoprotegerin as a Predictor of Coronary Artery Disease and Cardiovascular Mortality and Morbidity. J Am Coll Cardiol. 2010;55:2049-61; DOI:10.1016/j. jacc.2010.03.013.

8. Price PA, June HH, Buckley JR, Williamson MK. Osteoprotegerin inhibits artery calcification induced by warfarin and by vitamin D. Arterioscler Thromb Vasc Biol. 2001;21:1610-6; DOI:10.1161/hq1001.097102.

9. Vorkapic E, Kunath A, Wågsäter D. Effects of osteoprotegerin/TNFRSF11B in two models of abdominal aortic aneurysms. Mol Med Rep. 2018;18:41-8; DOI:10.3892/mmr.2018.8936. 
10. Clancy P, Oliver L, Jayalath R, Buttner P, Golledge J. Assessment of a serum assay for quantification of abdominal aortic calcification. Arterioscler Thromb Vasc Biol. 2006;26:2574-6; DOI:10.1161/01. ATV.0000242799.81434.7d.

11. Zauli G, Corallini F, Bossi F, Fischetti F, Durigutto P, Celeghini C, Tedesco F, Secchiero P. Osteoprotegerin increases leukocyte adhesion to endothelial cells both in vitro and in vivo. Blood. 2007;110:536-43 DOI:10.1182/blood-2007-01-068395.

12. Wen WW, Ning Y, Zhang Q, Yang YX, Jia YF, Sun HL, Qin YW, Fang F, Zhang M, Wei YX. TNFRSF11B: A potential plasma biomarker for diagnosis of obstructive sleep apnea. Clin Chim Acta. 2019;490:39-45; D0I:10.1016/j.cca.2018.12.017

13. Modi WS. CCL3L1 and CCL4L1 chemokine genes are located in a segmental duplication at chromosome 17q12. Genomics. 2004;83:735-8, DOI:10.1016/j.ygeno.2003.09.019.

14. Gäbel G, Northoff BH, Weinzierl I, Ludwig S, Hinterseher I, Wilfert W, Teupser D, Doderer SA, Bergert H, Schönleben F, Lindeman JHN, Holdt LM. Molecular fingerprint for terminal abdominal aortic aneurysm disease. Am Heart Assoc. 2017;6; DOI:10.1161/JAHA.117.006798.

15. Suviolahti E, Ge S, Nast CC, Mirocha J, Karasyov A, White M, Jordan SC, Toyoda M. Genes associated with antibody-dependent cell activation are overexpressed in renal biopsies from patients with antibody-mediated rejection. Transpl Immunol. 2015;32:9-17; DOI:10.1016/j. trim.2014.11.215

16. Hassan MI, Ahmad F. Structural diversity of class i MHC-like molecules and its implications in binding specificities. Adv. Protein Chem. Struct. Biol., vol. 83, Academic Press Inc.; 2011, p. 223-70; DOI:10.1016/ B978-0-12-381262-9.00006-9.

17. Sugimoto T, Sada M, Miyamoto T, Yao H. Genetic analysis on HLA loci in Japanese patients with abdominal aortic aneurysm. Eur J Vasc Endovasc Surg. 2003;26:215-8; DOI:10.1053/ejvs.2002.1912.

18. Otsuka A, Hanafusa T, Kono N, Tarui S. Lipopolysaccharide augments HLA-A,B,C molecule expression but inhibits interferon-gamma-induced HLA-DR molecule expression on cultured human endothelial cells. Immunology. 1991;73:428-32.

19. Mallis P, Michalopoulos E, Dinou A, Vlachou MS, Panagouli E, Papapanagiotou A, Kassi E, Giokas CS. Development of HLA-matched vascular grafts utilizing decellularized human umbilical artery. Hum Immunol. 2018;79:855-60; DOI:10.1016/j.humimm.2018.09.001.

20. El-Tayeb A, Qi A, Nicholas RA, Müller CE. Structural modifications of UMP, UDP, and UTP leading to subtype-selective agonists for P2Y2, P2Y4 and P2Y6 receptors. J Med Chem. 2011;54:2878-90; DOI:10.1021/ jm1016297.

21. Hasselhof V, Sperling A, Buttler K, Ströbel P, Becker J, Aung T, Felmerer G, Wilting J. Morphological and molecular characterization of human dermal lymphatic collectors. PLoS One. 2016;11; DOI:10.1371/journal. pone. 0164964 .

22. Hennigs JK, Lüneburg N, Stage A, Schmitz M, Körbelin J, Harbaum L, Matuszcak C, Mienert J, Bokemeyer C, Böger RH, Kiefmann R, Klose H. The P2-receptor-mediated Ca2+ signalosome of the human pulmonary endothelium - implications for pulmonary arterial hypertension. Purinergic Signal. 2019;15:299-311; DOI:10.1007/s11302-019-09674-1. 